\title{
Archéopages
}

Archéopages

Archéologie et société

$43 \mid 2016$

Médecines

\section{Au fil de Sirona... la déesse de Mediolanum/Mâlain}

Nouvelles données épigraphique et iconographique

Following Sirona...the goddess of Mediolanum/Mâlain. New epigraphic and iconographic data

Marie-Agnès Widehen et Michel Kasprzyk

\section{OpenEdition \\ Journals}

Édition électronique

URL : https://journals.openedition.org/archeopages/1847

DOI : 10.4000/archeopages. 1847

ISSN : 2269-9872

Éditeur

INRAP - Institut national de recherches archéologiques préventives

Édition imprimée

Date de publication : 1 novembre 2016

Pagination : 28-33

ISSN : 1622-8545

Référence électronique

Marie-Agnès Widehen et Michel Kasprzyk, "Au fil de Sirona... la déesse de Mediolanum/Mâlain », Archéopages [En ligne], 43 | 2016, mis en ligne le 01 décembre 2018, consulté le 03 juin 2021. URL : http://journals.openedition.org/archeopages/1847 ; DOI : https://doi.org/10.4000/archeopages.1847 


\section{Au fil de Sirona... la déesse de Mediolanum/Mâlain \\ Nouvelles données épigraphique et iconographique}

Marie-Agnès Widehen Inrap

Michel Kasprzyk Inrap, UMR 6298 «ArTeHis»
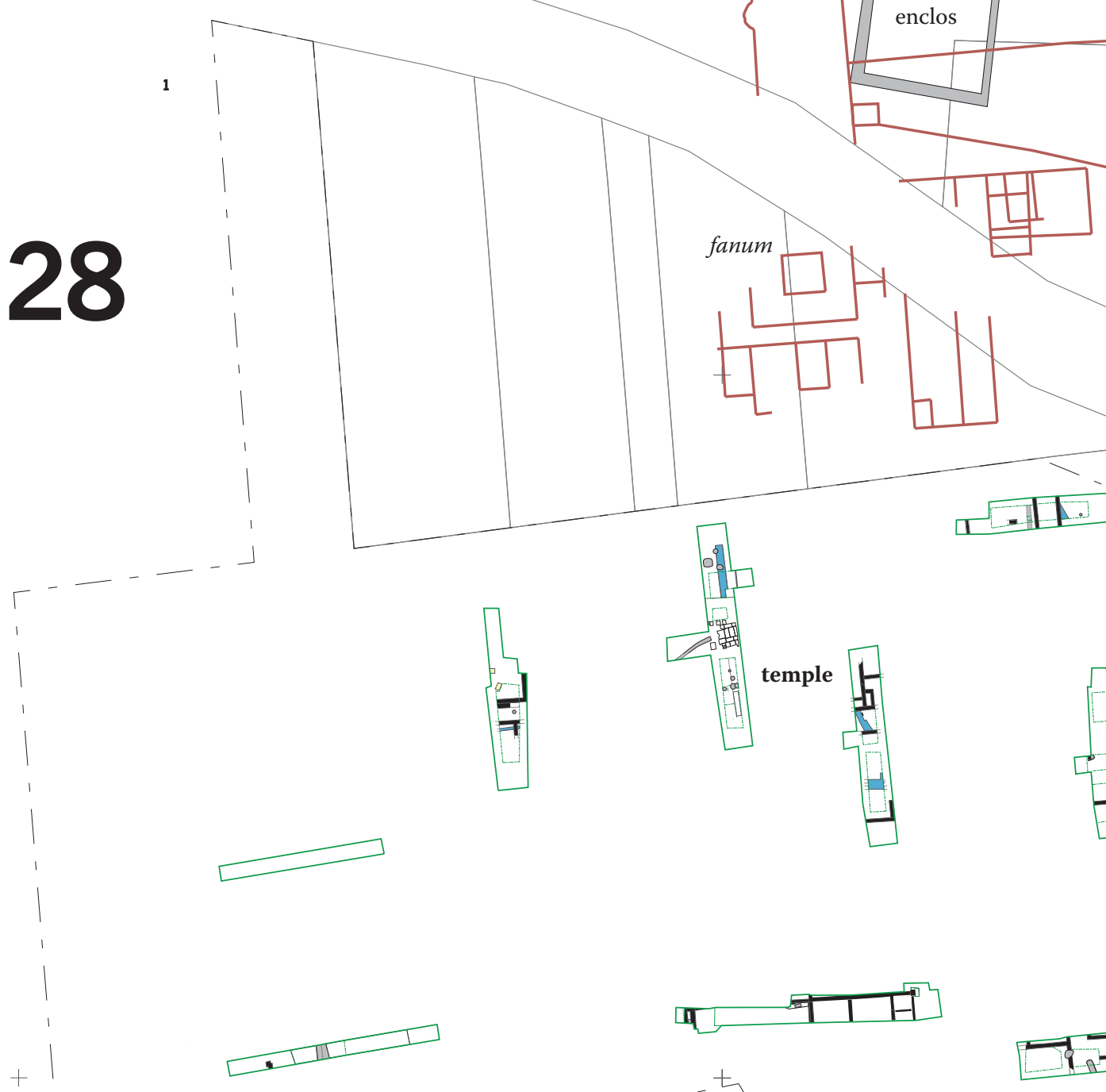

galerie?
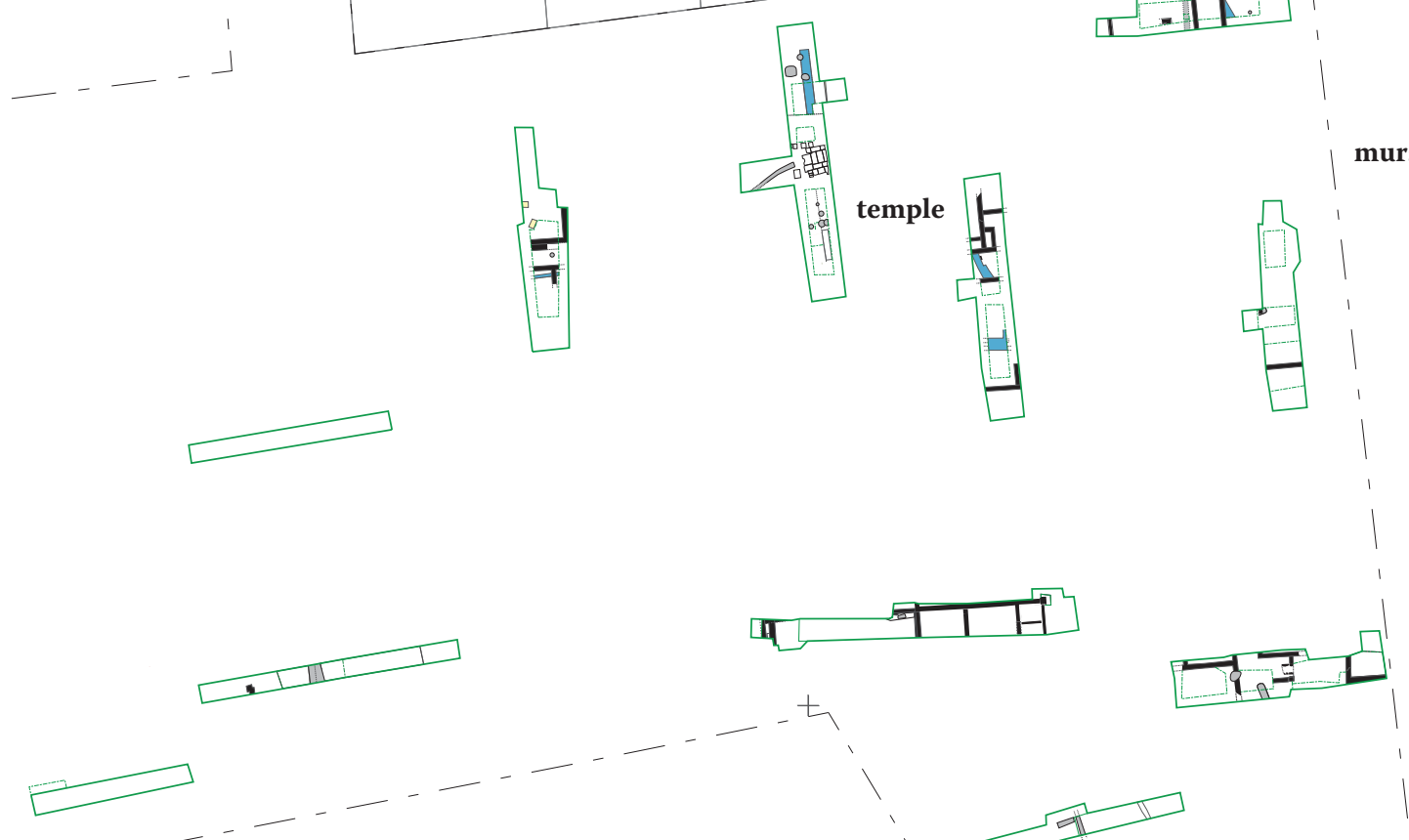
murs de clôture?
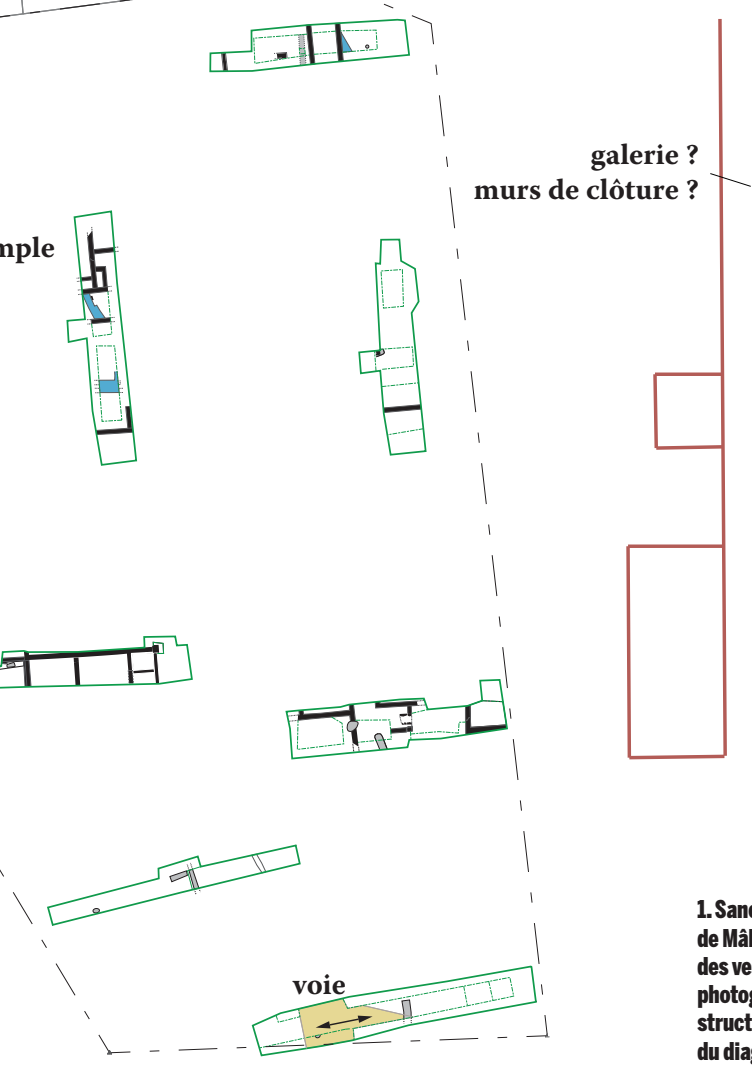
Le village de Mâlain, situé en Côte-d'Or, aux marges de l'Auxois, a fait l'objet de nouvelles investigations archéologiques en $2014^{1}$. La réalisation d'un diagnostic a offert l'opportunité de mieux connaître le sanctuaire occidental de l'agglomération antique. Deux découvertes lapidaires exceptionnelles et la mise au jour d'ex-voto anatomiques nous apprennent qu'au sein de ce complexe religieux, les fidèles s'adressaient au dieu Apollon et à la déesse Sirona pour des besoins de guérison.

\section{L'agglomération antique et le sanctuaire occidental}

L'agglomération de Mâlain/Mediolanum, située au sud de la cité des Lingons, est traversée par une voie reliant Dijon/Divio au site d'Alésia. Elle se développe de part et d'autre de la rivière Douix, affluent de l'Ouche. L'occupation gauloise, attestée à partir du milieu du $\mathrm{II}^{\mathrm{e}}$ siècle avant notre ère, n'est connue que sporadiquement. À l'est de la Douix, se développent des quartiers d'habitat et d'artisanat occupés de la fin de l'âge du Fer à la fin du III ${ }^{\mathrm{e}}$ siècle (Roussel, 1988). À l'ouest, le site, tel qu'il nous est connu, est caractérisé par la présence de plusieurs édifices publics. C'est au pied du Mont Chauvin qu'a été implanté le grand sanctuaire récemment diagnostiqué. Ce complexe religieux était jusquà présent documenté par des découvertes effectuées au XIX ${ }^{\mathrm{e}}$ siècle et par plusieurs clichés aériens réalisés par René Goguey. Les découvertes

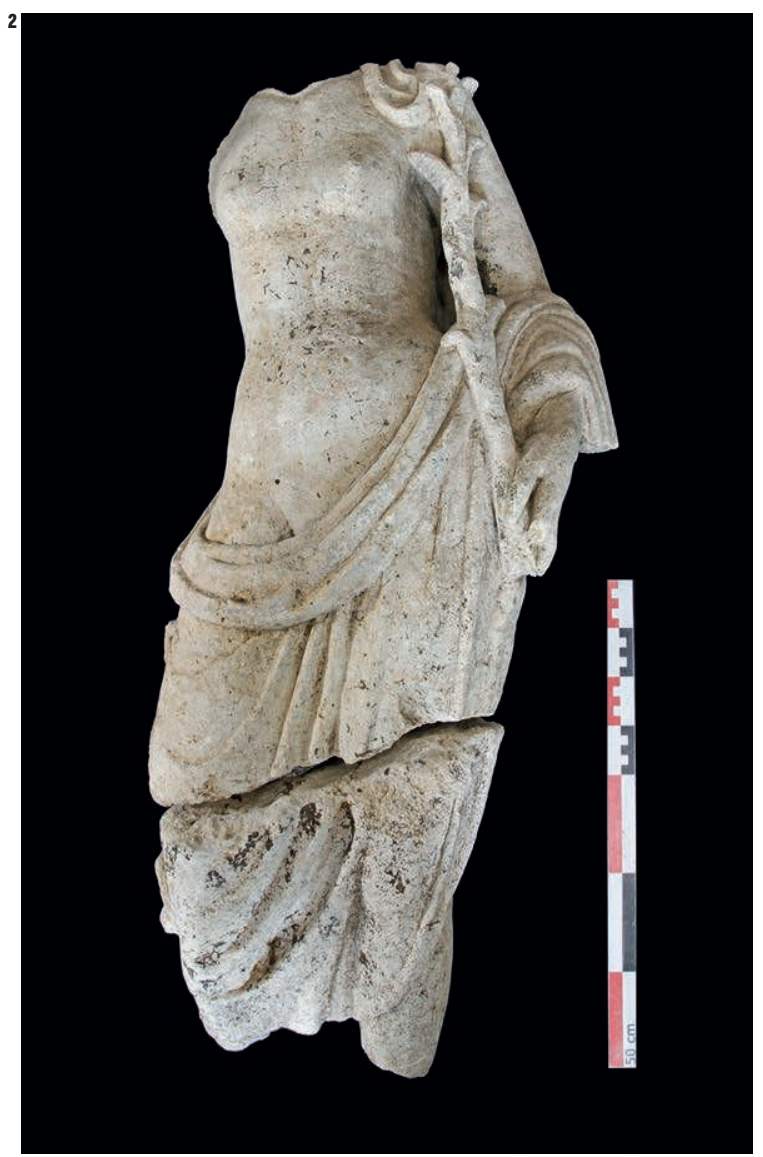

épigraphiques anciennes ont montré qu'en ce lieu étaient célébrés le dieu poliade Mars Cicolluis et sa parèdre Litavis/Bellona. L'existence d'un enclos fossoyé originel a été reconnue au nord tandis que, du plan des constructions romaines, se distinguent un temple de type fanum, ainsi que des murs de clôture ou de galerie pouvant correspondre au tracé oriental du péribole.

L'emprise de la zone diagnostiquée s'inscrit dans les limites du sanctuaire [ill. 1]. Le site est occupé de La Tène finale (à partir de 9o/80 avant notre ère) à la deuxième moitié du $\mathrm{III}^{\mathrm{e}}$ siècle. Les niveaux les plus anciens témoignent d'un environnement originel humide. Des structures attribuables à l'époque augusto-tibérienne (dallages en pierres brutes, fossés, trous de poteaux) ont été découvertes. À la fin du règne de Tibère ou sous le règne de Claude, le sanctuaire est monumentalisé et quatre canalisations souterraines précèdent les premières constructions maçonnées. L'une, comme nous avons pu le constater de manière vivante, est une adduction de source. Deux phases de réaménagements, la première se déroulant durant la seconde moitié $\mathrm{du} \mathrm{I}^{\mathrm{er}}$ siècle, la seconde au cours du $\mathrm{II}^{\mathrm{e}}$ siècle, probablement sous le règne d'Hadrien, peuvent être envisagées en l'état des données.

Au nord du terrain diagnostiqué, les vestiges architecturaux et la découverte de nombreuses offrandes accréditent l'idée que nous sommes au cœur d'une des aires cultuelles. Dans son dernier état, le temple qui y a été reconnu, de style classique si l'on en juge par la découverte d'un fragment de corniche corinthienne modillonnaire (premier tiers du $\mathrm{II}^{\mathrm{e}}$ siècle), semble intégrer un parcours hydraulique. En effet, d'un canal supposé, l'eau était évacuée par une conduite en partie souterraine dont le comblement a livré du mobilier daté des $\mathrm{II}^{\mathrm{e}}$ et $\mathrm{III}^{\mathrm{e}}$ siècles. Les niveaux hydromorphes anciens, le réseau de canalisation souterrain et les différents niveaux d'inondation constatés sur l'ensemble de la stratigraphie suggèrent que l'eau jouait un rôle particulier au sein du sanctuaire de Mâlain. Si aucun bassin en tant que tel n'est apparu aux endroits sondés, la célébration des divinités Apollon et Sirona conforte cette impression. Immédiatement au sud du bâtiment cultuel, sur une couche de destruction ayant notamment livré un autel votif dédié au dieu Apollon Mogetimarus, a été mise au jour la sculpture d'un personnage féminin dont un fragment, représentant des jambes, a été découvert plus à l'ouest où se trouvait également une base de statue épigraphe offerte à la divinité Sirona [ill. 2 et encadré]. Si l'on ne peut être totalement assuré, pour l'instant, que la base inscrite et le corps féminin appartiennent à la même œuvre, il y a tout lieu de penser que ces vestiges sont des représentations de la même divinité. Recouverte par la terre végétale, la base se tenait dans un espace a priori extérieur, composé d'un sol en grandes dalles calcaires associées à des
1. Diagnostic réalisé à Mâlain, Rue de Sercey, du 24 novembre au 16 décembre 2014, sou la direction de MarieAgnès Widehen, Inrap.

\begin{abstract}
dés de support. Ce dallage était encadré, au nord
\end{abstract}


et au sud, par un sol en concassé de calcaire blanc sur lequel ont été recueillis deux ex-voto anatomiques en alliage cuivreux ${ }^{2}$.

\section{Les images de Sirona}

La sculpture de Sirona découverte à Mâlain évoque de prime abord une Vénus ou une Nymphe et ne serait pas antérieure au $\mathrm{II}^{\mathrm{e}}$ siècle. Figurée debout, dans une pose un peu déhanchée selon le style classique gréco-romain, la divinité est chaussée de sandales. Un drapé dévoile légèrement son pubis puis, ramené dans le dos, réapparaît sur son épaule gauche où repose un végétal. La tige au port droit et aux feuilles alternes évoque un saule. Cette œuvre, qui représente la divinité à moitié dévêtue, nous renvoie au groupe de statuettes en bronze réunissant Sirona et Apollon citharède, découvert autrefois à Mâlain et daté du deuxième quart du $\mathrm{III}^{\mathrm{e}}$ siècle. Leurs corps se répondant par symétrie, les deux divinités y sont représentées debout sur un socle où se lit l'inscription THIRONETAPOLLO. Un serpent apparaît suspendu au poignet droit de la déesse (Rolley, 1988).

L'iconographie romaine offre des représentations variées de la déesse Sirona. À Hoscheid (Rhénanie-Palatinat), elle porte un diadème, un serpent est enroulé sur son avant-bras droit et elle maintient, à gauche, une coupe contenant des œufs (II ${ }^{\mathrm{e}}$ siècle) (Grenier, 1960).

2. Cf. article de Stéphanie Raux dans ce numéro. 3. Esculape est d'ailleurs honoré avec Jupiter, Apollon et Sirona sur la dédicace du centurion Publius Aelius Lucius découverte à Vienne (Autriche) $(A E$ 1957, 114). 4. Deux autres

figurations de la déesse provenant de Sainte-

Fontaine ont été interprétées comme des images d'Hygie

(Espérandieu, 1908-1922,

$\mathrm{V}, \mathrm{n}^{\circ} 4442$ et 4454 ).

5. Sur les trois autres

faces de l'autel découvert

à Vienne-en-Val, sont figurés Apollon tenant « un plectre énorme, qui paraît représenter des poissons (...) », Hercule et Minerve.

6. Mentionnons enfin un autel trouvé en 1865 à Bordeaux, dans

les fondements de la muraille romaine, sur lequel figurent les bustes de quatre divinités, dont peut-être Sirona tenant un sceptre de la main gauche, Mercure, Mars et Hercule (Espérandieu, 1908-0922, II, n ${ }^{\circ}$ 1077).

7. À Faimingen (Moselle) Apollon Grannus a eu également pour parèdre Hygie : Apollini Granno et Sanctae Hygiae [---]/ [M]at(ri) deum ipsorum pro salute Luci[ (CIL III, 5873).
À Alésia (Côte-d'Or), la base de son diadème est ceinturé d'épis ; elle tient un serpent de la main gauche et présente une patère de la main droite (De Cazanove et al., 2012). Sur un autel découvert à Baumberg (Bavière), la déesse a, comme attributs, deux épis de blé et sans doute une grappe de raisin (Robert, 1879). Toutes ces représentations, y compris une base de statue découverte à Trêves (Rhénanie-Palatinat) (Robert, 1879), montrent Sirona habillée d'une longue tunique. A contrario, la déesse de Mâlain apparaît par deux fois le torse nu, ce qui constitue une spécificité dans le corpus des œuvres bien identifiées de ce personnage divin.

\section{Une divinité guérisseuse}

Sirona, figurée en compagnie d'un serpent, est interprétée comme l'image gallo-romaine de la déesse de la santé Hygie, fille du dieu médecin Asklepios/Esculape ${ }^{\mathbf{3}}$. Le lien à l'animal est tel qu'une confusion entre les deux divinités s'installe dans le cas de figurations dépourvues d'inscription (Nagy, 1994). Il faut convenir que la déesse de Mâlain n'est pas sans rappeler deux sculptures identifiées comme des représentations d'Hygie. L'une a été découverte à Cologne : la divinité, qui porte un drapé à la disposition similaire, pose le pied gauche sur une tête de veau et un serpent, glissant le long d'un autel puis autour du bras du personnage, s'approche d'une coupe de fruits (Espérandieu, 1908-1922, VIII, n 6421). La deuxième a été mise au jour à Laneuvevilledevant-Nancy (Meurthe-et-Moselle) (Espérandieu, 1908-1922, VI, n 4695). Il est intéressant également de citer le personnage féminin du groupe statuaire provenant du sanctuaire de Genainville (Vald'Oise) qui, appuyé sur une urne renversée, siège aux côtés d'une déesse mère accompagnée d'un enfant ainsi qu'un dieu qui a pu être Apollon (Deyts, 1992, p. 110 ; voir également Espérandieu 1908-1922, II, n 1321).

Le serpent est symboliquement attaché au monde chtonien et aux concepts de renaissance et de fécondité. Incarnation du pouvoir de guérison, il semble également lié à l'eau (Green, 1992, p. 224). Il est un fait que la dimension aquatique de Sirona est indissociable de son rôle salutaire et nombre de monuments figurés ou de dédicaces, qui la montrent parfois en compagnie du dieu médecin Apollon, ont été mis au jour en des lieux votifs liés à l'eau : Hochscheid (Grenier, 1960), Alésia (De Cazanove et al., 2012), Luxeuil-LesBains (Lombard, 200o). Par ailleurs, à Flavigny (Cher), la dédicace a peut-être consacré une fontaine ou une source locale (Cravayat, Lebel, Thévenot, 1956). Au Mans, la base de statue, en remploi dans le mur de la ville, pourrait provenir du site des Jacobins où se trouvait un étang sacré (Chevet et al., 2014). L'eau de source comprise comme l'émanation de la divinité semble exprimée dans le traitement très schématique des cheveux du portrait de Sirona découvert à Sainte-Fontaine (Freyming-Merlebach, Moselle) (Espérandieu 1908-1922, V, n $\left.{ }^{\circ} 4470\right)^{4}$. Par le don de l'eau, indispensable à la croissance végétale, l'aura de Sirona semble également associée à la fertilité de la terre, à la fécondité et à la prospérité. Ainsi, sur un autel provenant du sanctuaire de Vienne-en-Val (Loiret), le personnage nourrissant un serpent et couronné d'un diadème triangulaire surmonté d'une boule, interprété par Gilbert-Charles Picard comme étant Sirona, porte une corne d'abondance (Picard, 1970, p 185, 190) ${ }^{5}$. Ce symbole, ainsi que les épis de blé qui accompagnent parfois la déesse des eaux, ne sont pas sans rappeler l'image de Déméter ${ }^{\mathbf{6}}$.

\section{Son ascendance gauloise}

Il faut admettre que l'identité gauloise de Sirona apparaît évanescente. Tout ce que nous connaissons de la déesse date en effet de l'époque romaine. Le plus ancien témoignage pouvant lui être rapporté, découvert à Wiesbaden (Hesse), remonterait au dernier quart du $\mathrm{I}^{\mathrm{er}}$ siècle de notre ère (CIL XIII, 7570), la plupart des documents se situant entre 150 et 250 de notre ère (Nagy, 1994). Dans la documentation iconographique, Sirona est la parèdre de l'Apollon musicien dont l'attribut est la lyre, symbole d'harmonisation. À Hoscheid, un griffon, animal fabuleux jouant un rôle dans la destinée des âmes (Compatangelo-Soussignan, 2012, p. 25, 33) est également sculpté aux côtés du dieu. D'autre part, parmi les inscriptions, six d'entre elles associent Sirona à Apollon Grannus ${ }^{7}$. Mais bien qu'apparaissant en couple, Sirona, parfois nommée Sancta Sirona - ou de manière 
8. Alise-Saint-Reine (Côte-d'Or) (CIL XIII 11243), Ihn ( $A E$ 1994, 1256 et $A E$ 1994, 1257), Saint-

Avold/Freyming (Moselle) (CIL XIII 04498), Trêves

(Allemagne) (CIL XIII o3662).

9. Apollini / Granno / Dianae / [s]anct(a)e

Siron(a)e (AE 1992, 1304).

La ligne en question a fait

l'objet d'une rectification.

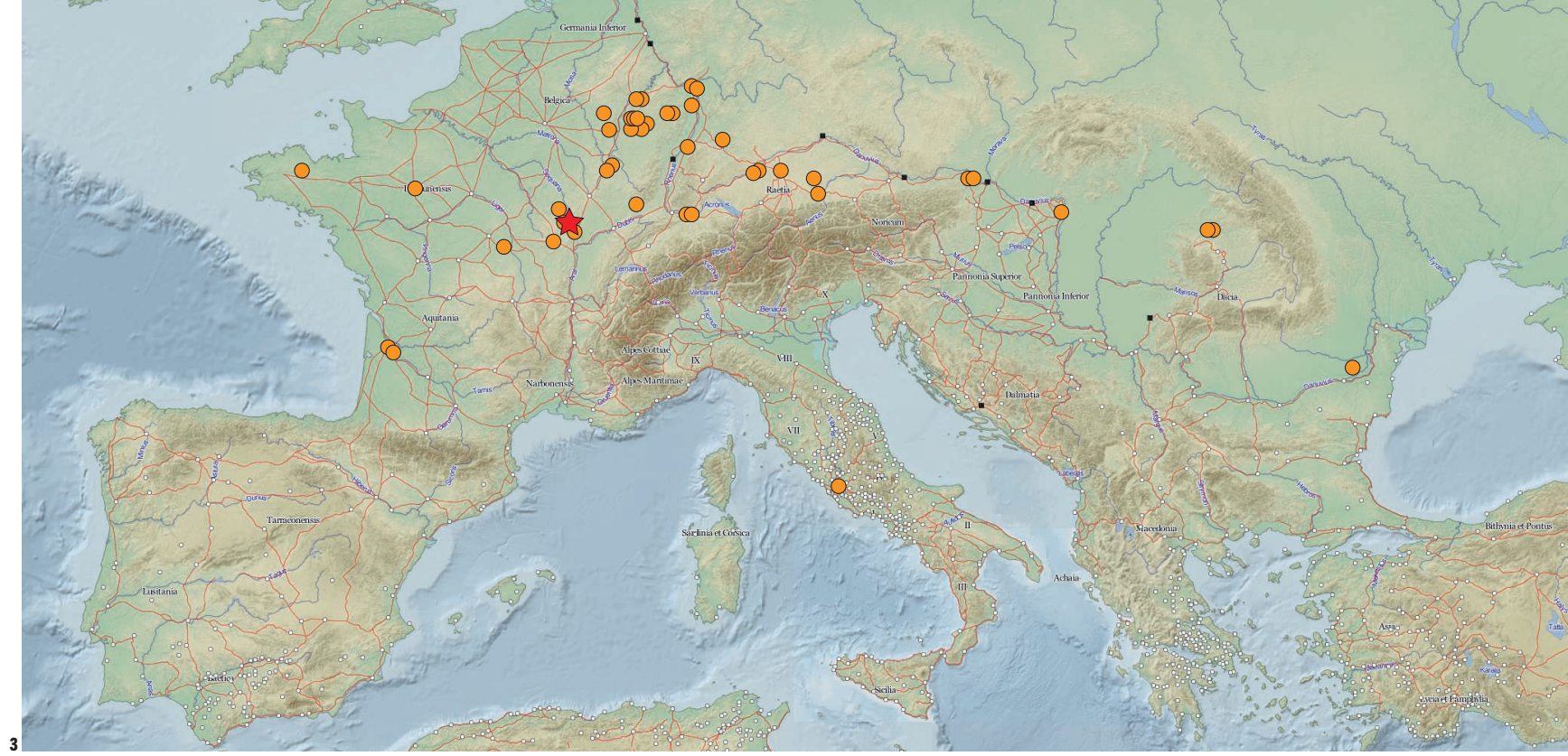

inédite Sirona Sivelia au Mans (Sarthe) - n'en est pas moins caractérisée par une identité marquée.

La transcription de son nom, usant de l'affriquée «ts » (Th ou $Đ)$, indique l'origine indigène de la déesse ${ }^{\mathbf{8}}$. Son appellation comprend le suffixe féminin d'individualisation -ona rattaché hypothétiquement au mot ster-ou stir-qui, dans la sémantique indo-européenne, signifierait étoile (De Bernardo Stempel, 2013, p. 80 ; Hofeneder, 2013, p. 107-108). Sirona serait ainsi une déesse lumineuse, pendant du dieu solaire Apollon. Par ailleurs, son assimilation avec Diane sur l'inscription d'Augsbourg (Bavière) a conduit à envisager un rapprochement de cette divinité avec la lune ${ }^{9}$ (De Bernardo Stempel, 2013, p. 8o). Il faut néanmoins reconnaître que la dimension céleste de Sirona apparaît éloignée de son schéma iconographique tourné vers le monde chtonien/ aquatique et que nous nous heurtons ici à notre ignorance du récit mythologique.
D'une autre manière, son origine celtique semble illustrée par la carte de répartition des documents épigraphiques la mentionnant. Parmi les dédicaces à la déesse, vénérée de l'Aquitaine à la Dacie, on constate en effet une plus grande concentration au nord des Alpes : dans l'est de la Gaule Belgique, en Germanie supérieure ainsi qu'en Rhétie [ill. 3]. En pays Lingon et Mandubien, Sirona est honorée dans le sanctuaire occidental de Mâlain et dans le sanctuaire d'Apollon Moritasgus à Alésia. Sa présence au sanctuaire des Bolards (Nuits-Saint-Georges), en pays éduen, reste pour l'heure soumise à caution $(A E$ 1994, 1225). Au sanctuaire de confins de Mediolanum, sont également célébrées les divinités Mars Cicolluis, Litavis, Bellona et probablement Apollon Mogetimarus. Les théonymes indigènes de ces divinités révèlent leur caractère topique et il est possible que la déesse Sirona ait été invoquée à Mediolanum dès l'époque gauloise.

\section{Références bibliographiques}

AE : L'année épigraphique, Paris, 1988-

César, De Bello gallico, Paris, Presses universitaires de France, 1974.

CIL : Corpus Inscriptionum Latinarum, Berlin, 1895

ESPÉRANDIEU E., 1908-1922, Recueil général des basreliefs, statues et bustes de la Gaule romaine, tomes $I I, V, V I, V I I I$, Paris, Imprimerie Nationale.

Chevet P., Raux S., et al., 2014, «Un étang sacré à Vindinum/Le Mans (Sarthe) », Gallia, 71.2, p. $125-162$

Compatangelo-Soussignan R., 2012, « Apollon en Cisalpine : philosophie, religion et idéologie des élites romaines au $\mathrm{II}^{\mathrm{e}}$ siècle av. n.è. », Mélanges de l'École française de Rome - Antiquité, 124-1, p. 2-37.

Cravayat P., Lebel P., Thévenot E., 1956, «Une dédicace à Apollon et Sirona découverte à Flavigny (Cher) », Revue archéologique de l'Est, t. VII, p. 318-331.

De Bernardo Stempel P., 2013, « Celtic and Other Indigenous Divine Names Found in the Italian Peninsula ", in Hofeneder A., De Bernardo Stempel P. (éd.), Théonymie celtique, cultes, interpretatio, Vienne (Mitteilungen der Prähistorischen Kommission 79), p. 73-96.
De Cazanove O., Barrière V., Creuzenet F., et al. 2012, « Le lieu de culte du dieu Apollon Moritasgus à Alésia. Phases chronologiques, parcours de l'eau, distribution des offrandes ", in De Cazanove O. MÉniel P. (dir.), Étudier les lieux de culte de Gaule romaine, Montagnac, Monique Mergoil (Archéologie et Histoire romaine, 24), p. 95-121.

Deyts S., 1992, Images des Dieux de la Gaule, Paris, Errance, $159 \mathrm{p}$

Green M., 1992, Animals in Celtic Life and Myth, Londres, New-York, Routledge, 283 p.

Grenier A., 1960, « Le temple d'Apollon et de Sirona à Hochsheid », in Manuel d'archéologie gallo-romaine: quatrième partie, les monuments des eaux; Villes d'eau et sanctuaires de l'eau, Paris, A. et J. Picard, p. $884-893$

Hofeneder A., 2013, « Apollon Grannos Überlegungen zu Cassius Dio 77, 15,5-7 », in Hofeneder A. DE Bernardo Stempe P. (éd.), Théonymie celtique, cultes, interpretatio Vienne (Mitteilungen der Prähistorischen Kommission 79), p. 101-111.
LOMBARD, 2000, «Le monument de Luxeuil-les-Bain consacré à Apollon et à Sirona », in WALTer H. (dir.), La sculpture d'époque romaine dans le nord, dans l'est des Gaules et dans les régions avoisinantes: acquis et problématiques actuelles, Besançon, Presses universitaires franc-comtoises (coll. Annales littéraires art et archéologie, 45), p. 179-202.

NAGY A. M., 1994, « Sirona », in Lexicon Iconographicum Mythologiae Classicae, tome VII, Zürich/München, p. 779-781.

PICARD G., 1970, «Les fouilles de Vienne-en-Val (Loiret) », Comptes rendus des séances de l'Académie des Inscriptions et Belles-Lettres, $114^{\mathrm{e}}$ année, $\mathrm{n}^{\circ} 2$, p. 176-191.

RAEPSAET-CHARLIER M.-T., 2013, "Alésia et ses dieux : du culte d'Apollon Moritasgos à l'appartenance civique des Mandubiens à l'époque gallo-romaine ", L'Antiquité Classique, 82, 2013, p. 165-194.

Robert C., 1879, «Sirona », Revue Celtique, t. IV, p. $133-144$

Rolley C., 1988, « Les statuettes en bronze. La Trouvaille des Champs-Marlots : Statuettes inscrites du III ${ }^{\mathrm{e}}$ siècle ", in Roussel L. (dir.), Mediolanum, une bourgade gallo-romaine. 20 ans de recherches archéologiques, Dijon, Musée archéologique, p. 287-292.

Roussel L. (dir.), 1988, Mediolanum, une bourgade gallo-romaine. 20 ans de recherches archéologiques, Dijon, Musée archéologique, 324 p. 


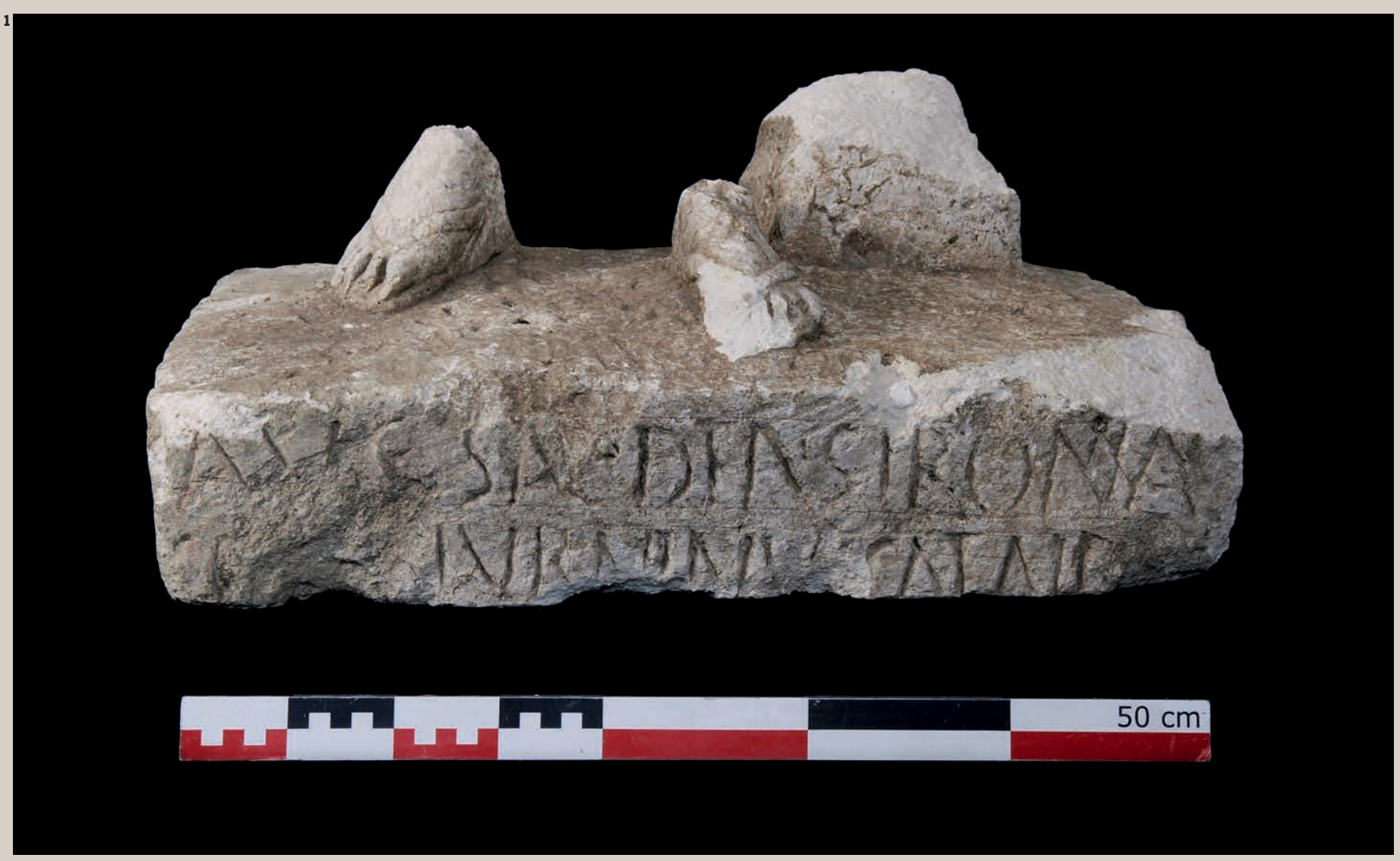

\section{Nouvelle}

documentation épigraphique à Mâlain

Les deux inscriptions trouvées à Mâlain viennent documenter le panthéon local d'une importante agglomération secondaire située aux confins des cités des Lingons et des Fiduens. Flles confirment de manière remarquable l'existence d'un culte rendu à Apollon et Sirona, qui était déjà attesté par un groupe de deux statuettes en bronze sur un même socle, trouvé à Mâlain (aux Champs Marlots) en 1977 et portant l'inscription Thiron(a) et Apollo (A.E, 1994, 1227).

Bien que l'on soit tenté d'associer les deux témoignages en considérant Sirona comme parèdre d'Apollon, il faut souligner que les deux documents sont de nature différente (un probable autel et un socle de statue) et que les deux divinités ne sont pas citées conjointement dans les dédicaces elles-mêmes.
La dédicace à Sirona est gravée sur un socle de statue en calcaire où seuls les pieds, chaussés de sandales, et le départ d'un autel (?) sont conservés. Les angles supérieurs sont brisés et la base du socle est en mauvais état d'où de nombreuses lacunes sur la partie inférieure des lettres [ill. 1]. Le texte est réparti sur deux lignes, un gros éclat entame la partie gauche du champ épigraphique. Les lettres en capitales apparaissent peu soignées à frustes, en dépit de traces de réglures très visibles ( $\mathrm{F}, \mathrm{S}, \mathrm{T}$ étroits ; hastes des $\mathbf{N}$ obliques, d'où des confusions possibles avec $\mathrm{V}$ ).

AVG SAC DEA SIRONA / S[---]TVRNINV[-] SATAI+

Aug(usto) sac(rum), dea(e) Sirona(e), / Saturninus Satair[---]?

Consacré à l'Auguste et à Dea Sirona, (...) Saturninus Satair(o) ?...

Dans la documentation épigraphique, qui comprend une quarantaine d'inscriptions réparties de l'Aquitaine à la Dacie, Sirona peut être la parèdre d'Apollon, mais est le plus souvent honorée seule. L'inscription de Mâlain, offerte vraisemblablement par un pérégrin, associe son culte à celui de l'empereur comme sur l'inscription découverte à Fanum Martis/Corseul 
chez les Coriosolites (CIL XIII, 3143). Il en va de même sur cinq autres dédicaces où elle apparaît également en compagnie d'Apollon : chez les Trèvires à Vicus Beda/Bitburg (CIL XIII, 4129) et à Augusta Treverorum/Trèves (Robert, 1879); chez les Vindélices à Lauingen en Rhétie (CIL III, 1 1903) ; chez les Bituriges Cubes à Flavigny ( $A \mathbb{E} 1958$, 193) et à Grossbottwar dans les champs décumates (CIL XIII, 6458).

L'autel en calcaire est quant à lui brisé à la base et une moulure supérieure est à peine conservée. Les angles supérieurs droits et gauches sont brisés [ill. 2]. Le texte est gravé sur six lignes, la première étant séparée des suivantes par une moulure. L'inscription est lacunaire sur la première ligne, à gauche et à droite, ainsi que sur la deuxième ligne à droite. Le tracé des lettres, effectué en capitales carrées, est soigné. Si ce n'est sur la première ligne, des traces de réglures sont très visibles.

[AVG] SA[C] / APOL $<$ L $>$ IN[I] / MOGETIMR / DURAMA / DUDRI*FI / V*S*L*M

[Aug(usto)] sa[c(rum)] / Apollini / Mogetimar(o) / Durama / Dudri fi(lius ou -a) ? / v(otum) s(olvit) l(ibens) m(erito).

Consacré à l'Auguste et à Apollon Mogetimarus Durama, fille (?) de Dudrius, s'est acquittée de son vœu, de bon gré, comme il se doit.

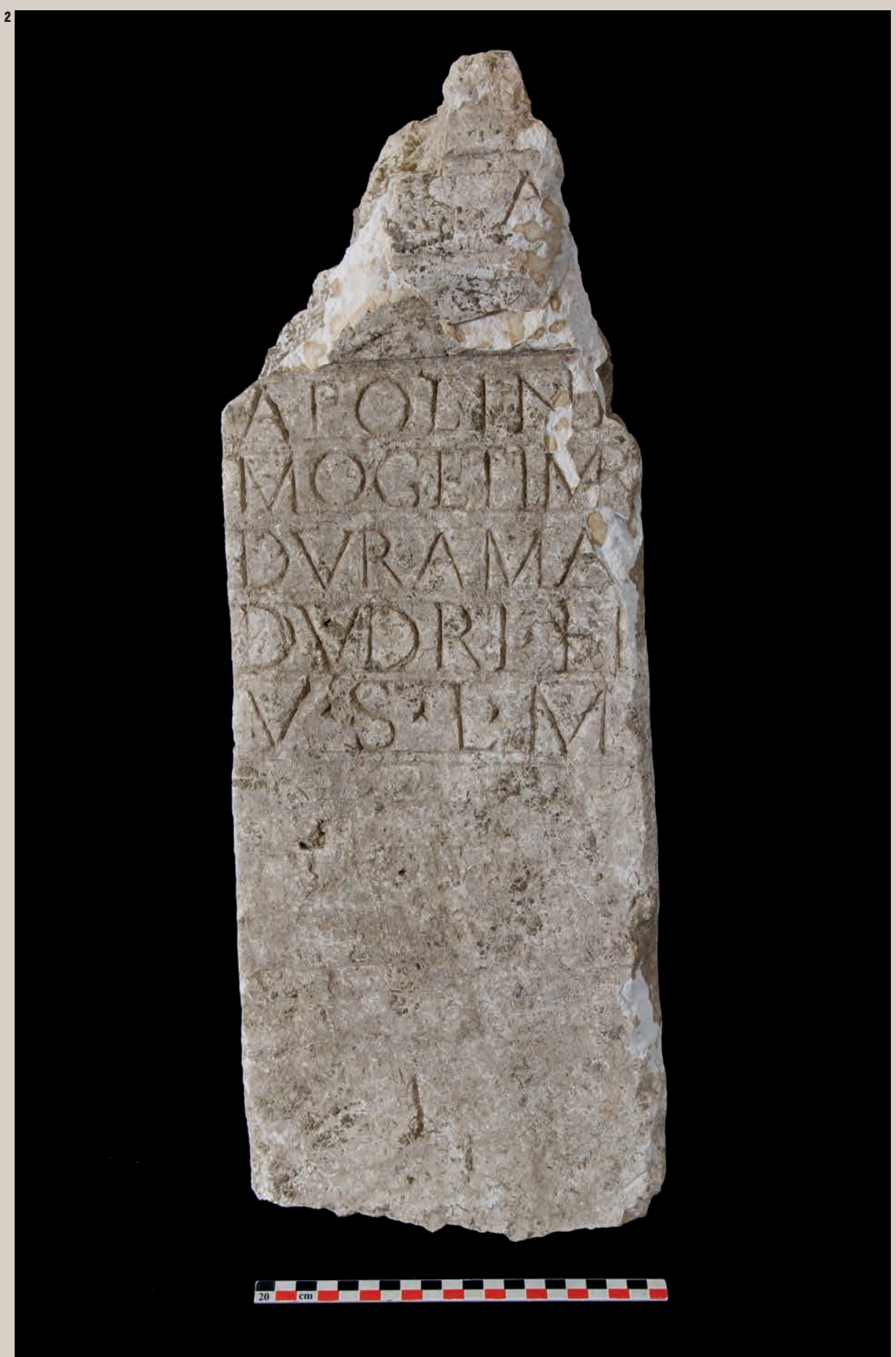

Cette dédicace à Apollon Mogetimarus, formulée par une personne de statut pérégrin, nous révèle une nouvelle appellation régionale du dieu.

Les divinités vénérées dans les sanctuaires de guérison proches d'Essarois et d'Alésia comportent également le nom latin Apollon auquel est apposée une épiclèse gauloise : Apollon Vindonnus (CIL XIII, 56445647) et Apollon Moritasgus (CIL XIII, 11240-1 1241). À propos du dieu d'Alésia, Marie-Thérèse RaepsaetCharlier relève que le nom Moritasgos est porté par un chef sénon durant la guerre des Gaules (César, BGV, 54)
(Raepsaet-Charlier, 2013,p. 183). L'épiclèse de Mâlain fait écho au nom de divinité Dinomogetimaro connu en Narbonnaise à Béziers (CIL XII, 42 18) Flle serait composée des mots mogeto" puissant » et *-maros " grand ". Mogetimarus est par ailleurs porté comme nom de personne à Riez (Narbonnaise) (CIL XII, 378) et à Virunum / Zollfeld (Norique) ( $A E 1993,1245$ ) et pourrait ainsi également être employé comme théophore. 